\title{
Carcinoma epidermoide sobre un divertículo de Zenker
}

\author{
D. MORALES GARCÍA, D. CASANOVA RITUERTO, M. GARCÍA DE POLAVIEJA, \\ I. G. RODILLA*, A. NARANJO GÓMEZ
}

Servicio de Cirugía General y del Aparato Digestivo. *Servicio de Anatomía Patológica. Hospital Universitario Marqués de Valdecilla. Santander. Cantabria.

EPIDERMOID CARCINOMA ARISING IN ZENKER'S DIVERTICULUM

\section{RESUMEN}

El carcinoma es una complicación rara, pero posible, de los divertículos faringoesofágicos. Se presenta el caso de un paciente de 49 años intervenido quirúrgicamente por un divertículo de Zenker en cuyo interior se descubrió la presencia de un carcinoma epidermoide. Dado que se trata de una complicación muy poco frecuente de esta patología, se revisa la literatura y se discuten las opciones terapéuticas.

PALABRAS CLAVE: Divertículo de Zenker. Divertículo faringoesofágico. Carcinoma epidermoide.

\begin{abstract}
Carcinoma is a rare but recognized complication of pharingoesophageal diverticulum. We report the case of a 49-year-old man operated for a Zenker's diverticulum with an epidermoid carcinoma arising in the diverticulum. Since carcinoma is an uncommon complication of this pathology, we review the literature and dicuss the therapeutic options.
\end{abstract}

KEY WORDS: Zenker's diverticulum. Pharingoesophageal diverticulum. Epidermoid carcinoma.

Morales García D, Casanova Rituerto D, García de Polavieja M, Rodilla IG, Naranjo Gómez A. Carcinoma epidermoide sobre un divertículo de Zenker. An Med Interna (Madrid) 2002; 19: 27-30.

\section{INTRODUCCIÓN}

El divertículo de Zenker se origina en la unión faringoesofágica, habitualmente en la parte posterior de la línea media, sobresaliendo entre las fibras oblicuas de los músculos constrictores de la faringe, inmediatamente por encima de las fibras transversas del músculo cricofaringeo (1-3) y representa la forma más frecuente de divertículo esofágico sintomático (4-10).

La descripción del divertículo faringoesofágico fue hecha inicialmente por Ludlow (11) en 1769, aunque son Zenker y Von Ziemssen quienes en 1874 hacen una revisión exhaustiva del mismo (12).

Su manifestación clínica más frecuente es la disfagia orofaringea y el diagnóstico se basa fundamentalmente en el esofagograma baritado (13).

Su tratamiento es quirúrgico. Fue Wheeler (14) en 1886 quien realizó la primera extirpación quirúrgica con éxito de un divertículo faringoesofágico, existiendo múltiples opciones terapéuticas en la actualidad $(1,13,15-19)$.

Las principales complicaciones del divertículo son las respiratorias tales como ronquera, asma, neumonitis o abscesos pulmonares que pueden incluso conducir a la muerte del paciente (4,20-24). Sin embargo, la degeneración maligna del divertículo es una complicación posible, pero muy poco frecuente con una incidencia que puede oscilar entre el $0,5 \%$ y el $1,5 \%$, que la mayoría de las veces pasa desapercibida de manera preoperatoria y cuyo tratamiento es controvertido $(25,26)$.

\section{CASO APORTADO}

Paciente varón de 49 años, sin antecedentes de interés, con historia de cuatro meses de evolución consistente en disfagia alta para sólidos y regurgitación. La exploración física y los análisis complementarios fueron normales. El paciente no refería astenia ni anorexia. Pérdida de peso no cuantificada pero mínima a pesar de la disfagia. Se realizó tránsito esófagogastroduodenal objetivándose una imagen sacular localizada en tercio superior de esófago con un tamaño de 5 centímetros compatible con un divertículo de Zenker (Fig. 1). No fue posible realizar una esofagoscopia al existir una estenosis en la boca de Killian. Con el diagnóstico de divertículo de Zenker, el paciente fue intervenido quirúrgicamente a través de una cervicotomía lateral izquierda, practicándose una

Trabajo aceptado: 8 de Mayo de 2000

Correspondencia: Dr. Dieter José Morales García. Hospital Universitario Marqués de Valdecilla. C/ Avda. de Valdecilla s/n. 39008 Santander. Cantabria. 
resección del divertículo y una miotomía del músculo cricofaringeo. El postoperatorio cursó sin incidencias y el paciente fue dado de alta una semana después de la intervención quirúrgica. El estudio anatomopatológico de la pieza fue informado como fragmento de tejido en forma de divertículo ocupado por carcinoma epidermoide infiltrante (Fig. 2). Se reevaluó de nuevo al paciente y se realizó una esofagogastroscopia que demostró la existencia de un esófago de calibre y mucosa normales, sin alteraciones en la zona de sutura. El paciente fue remitido al servicio de radioterapia y tratado mediante telecobaltoterapia en 3 campos con una dosis media de 5000 cGy. Cuatro años después de la intervención, el paciente esta asintomático.

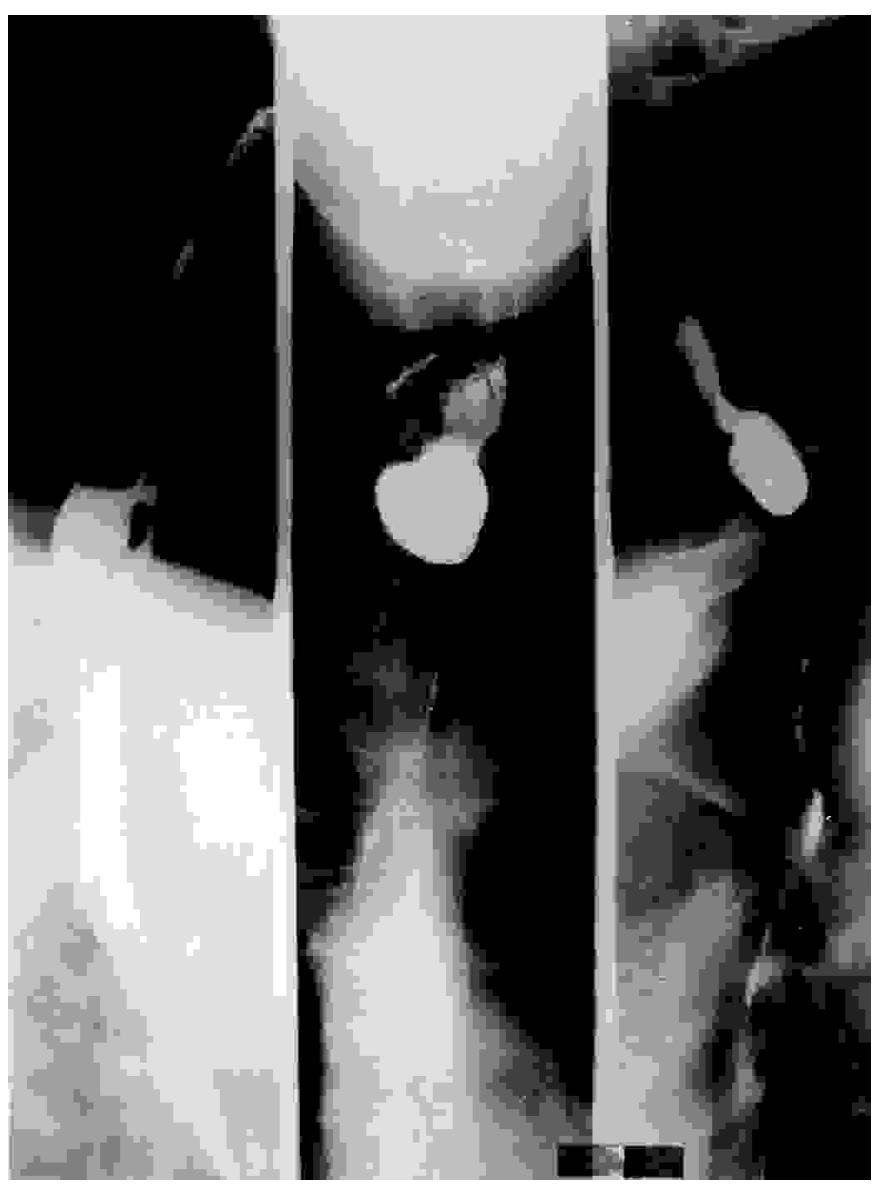

Fig. 1. Tránsito baritado esofagogastroduodenal en el que se observa una imagen en esófago compatible con un divertículo de Zenker. No aparecen datos sugestivos de malignidad.

\section{DISCUSIÓN}

La malignización de un divertículo de Zenker es una complicación extremadamente infrecuente. El primer autor que lo describió fue Schlesinger (27) (citado por Halstead en 1903) pero no es hasta 1927 cuando Vinson (28) comunica el primer caso de la literatura inglesa y desde entonces, han sido publicados menos de 50 casos.

En 1987, Bowdler y Stell (25) realizan una revisión de los 38 casos publicados hasta ese momento en la literatura inglesa, observando que esta patología afecta fundamentalmente a varones con una edad media superior a los 50 años. La inciden-



Fig. 2. Carcinoma epidermoide infiltrante con abundante componente inflamatorio en el margen de invasión y notable pleomorfismo nuclear.

cia de esta complicación se sitúa posiblemente entre un 0,5\% y el $1,5 \%(25,26)$ variando según las series revisadas, y disminuye en los estudios en los que el número de pacientes es alto. Wychulis (29) en 1969 describió 3 casos sobre una serie de 961 pacientes con una incidencia del $0,3 \%$ mientras que otros autores como Hoover (30) refiere 2 casos en una serie de 185 pacientes con una incidencia del $1,1 \%$ e incluso en series muy amplias el número de casos descritos es nulo (31).

La etiopatogenia de la degeneración maligna del divertículo no esta del todo aclarada. Se han propuesto una serie de factores predisponentes: por un lado estarían aquellos divertículos de larga evolución que tendrían una mayor tendencia a la degeneración maligna. La mayoría de los autores refieren una duración media de los síntomas en estos casos superior a los 11 años $(26,29,32-34)$ en pacientes varones y a partir de la quinta década de la vida, sin embargo hay casos descritos, como el nuestro, en el que la duración de los síntomas ha sido solo de meses $(25,35,36)$. La irritación crónica y la subsiguiente inflamación que se producen sobre el divertículo por la continua presencia de alimentos retenidos así como el trauma al que se ve sometido el divertículo para poderse vaciar han sido también propugnadas como situaciones predisponentes para su malignización $(23,25,26,32,37)$. Es frecuente que la malignización ocurra sobre una mucosa con cambios en la maduración celular y zonas de leucoplasia $(23,25,26,38)$.

El tipo histológico de tumor más frecuentemente encontrado en estos casos es el carcinoma epidermoide $(23,25)$, con distintos grados de diferenciación, e incluso se han descrito casos de carcinoma in situ, que es una forma aún más infrecuente $(26,36,39,40,41)$. También han sido descritas tumoraciones de tipo benigno en el interior de estos divertículos (33).

La forma de presentación clínica de los pacientes con un carcinoma sobre divertículo es similar a aquellos que presentan un divertículo no complicado: disfagia orofaringea, regurgitaciones, perdida de peso, masa cervical, tos nocturna, aunque en ocasiones como el caso de los ancianos, las complicaciones de tipo respiratorio pueden ser las únicas manifestaciones $(1,26)$ aunque existen algunas propias de esta complicación como es la acentuación repentina de alguna de ellas y especialmente de la disfagia, que puede llegar a convertirse en afagia (25). Lieber- 
son (38) y Fisher (42) describen la presencia de restos sanguinolentos en los alimentos regurgitados así como la hemoptisis, como formas de presentación del divertículo malignizado. El dolor no es un síntoma típico del divertículo de Zenker, pero la aparición del mismo así como su acentuación progresiva son indicativos de malignización $(39,43)$.

En relación con el diagnóstico, una radiografía simple puede orientarnos cuando muestra un ensanchamiento sospechoso del espacio retrofaringeo $(44,45)$, pero son el esofagograma baritado y la esofagoscopia, las principales pruebas diagnósticas. El esofagograma baritado, además de poner de manifiesto la existencia de un divertículo, puede proporcionar datos a cerca de su malignización como es la imagen de un defecto de repleción que permanece constante. Los restos alimenticios y la presencia de aire en el divertículo pueden simular esta imagen, pero típicamente se localizan en la parte superior del divertículo, mientras que el defecto de repleción producido por el tumor se localiza en el fundus o en la pared lateral del divertículo, generalmente en los dos tercios inferiores $(25,26,33)$, aunque hay casos de carcinomas descritos sobre el cuello del saco diverticular $(42,46)$. Para algunos autores, la esofagoscopia constituye una prueba diagnóstica esencial, exista sospecha de malignidad o como paso previo a la cirugía del divertículo no complicado ya que permite visualizar y si es necesario, biopsiar, la mucosa del mismo $(23,25,26,29,33)$ opinión que nosotros también compartimos pero que en nuestro caso no fue posible realizar preoperatoriamente. Otros autores sin embargo consideran que esta prueba tiene poca utilidad diagnóstica y puede incluso perforar la pared del divertículo $(13,20,32)$. Jorgensen y cols. (26) opinan que la tomografía axial computarizada y la resonancia nuclear magnética podrían jugar un papel en el diagnóstico de extensión de estos tumores, aunque reconocen la escasa experiencia que hay al respecto. En cualquier caso, hay que tener en cuenta que la mayoría de las veces el diagnóstico de estos tumores se hace incidentalmente durante la cirugía o por el patólogo al estudiar la pieza $(25,35)$.

En relación con el tratamiento y dado que gran cantidad de los casos proceden de aportaciones individuales, la controversia es importante y es difícil determinar un método óptimo aunque parece que es la cirugía la que permite una actitud terapéutica definitiva. Si tenemos en cuenta que la mayoría de las veces el diagnóstico de cáncer se realiza de manera postoperatoria, evaluando retrospectivamente el tratamiento, un importante número de autores realizan una diverticulectomía simple asociada o no a radioterapia, que en el caso de que el tumor este limitado al cuerpo del divertículo sin afectar al cuello, puede ser curativa $(23,25,26,34)$. Cuando el tumor infiltra o sobrepasa el cuello diverticular o invade estructuras vecinas, algunos autores proponen tratamientos más agresivos con resecciones extensas del tipo faringolaringuectomía, aunque en estos casos, el pronóstico de los pacientes es infausto, con una supervivencia actuarial menor del $10 \%$ a los cinco años (25).

El papel de la radioterapia en este tipo de tumores también es muy discutido. Parece claro que su utilidad como tratamiento único, es decir, no asociada a cirugía, es escaso o nulo (25) y tendría solamente valor como tratamiento paliativo en caso de tumores inoperables. Cuando se dispone de un diagnóstico preoperatorio, la radioterapia podría ser útil con intención citorreductora (33) e incluso asociada a quimioterapia (26). Su valor como tratamiento coadyuvante de la cirugía también esta muy discutido. Para algunos autores no hay suficientes evidencias de que aporte beneficios al tratamiento quirúrgico (26). Sin embargo, para otros autores, entre los que nos incluimos, aportaría beneficios y debería de aplicarse al menos de manera postoperatoria como tratamiento coadyuvante de la cirugía tras una diverticulectomía simple en la que el tumor no sobrepasase el cuello del divertículo, pudiendo ser incluso válida en casos límite como los tumores con afectación proximal o del cuello diverticular, frente a opciones quirúrgicas más agresivas $(23,25)$.

Como consideraciones finales, insistir en que el tratamiento del divertículo de Zenker debe de ser quirúrgico y precoz para evitar su posible malignización. Respecto al diagnóstico pensamos que la esofagoscopia constituye un método de diagnóstico preoperatorio muy importante y a pesar de sus riesgos debe intentarse de manera sistemática. En relación con el tratamiento del divertículo malignizado, la diverticulectomía simple asociada a radioterapia constituye una opción terapéutica adecuada incluso en los casos límite permitiendo supervivencias prolongadas como en nuestro caso y representando una opción válida frente a grandes resecciones quirúrgicas que llegan a ser mutilantes para el paciente y que no mejoran su expectativa de vida de manera global.

\section{Bibliografía}

1. Molins L, Lluis J, Galofré M. Divertículo de Zenker. Manifestaciones clínicas y tratamiento. Rev Esp Enferm Dig 1992; 81: 313-5.

2. Ellis FH. The management of Zenker's diverticulum: Cricofaringeal myotomy. In: Kittle CF. Current controversies in thoracic surgery. Philadelphia. WB Saunders, 1986; 10-4.

3. Venturi M, Bonavina L, Colombo L, Antoniazzi L, Bruno A, Mussini E, Peracchia A. Biochemical markers of upper esophageal sphincter compliance in patients with Zenker's diverticulum. J Surg Res 1997; 70: 46-8.

4. Payne WS. Diverticula of the esophagus. In: Payne WS, Olsen AM. The Esophagus. Philadelphia: Lea and Febiger, 1974; 207-223.

5. Ellis FH. Disorders of the esophagus in the adult. In: Sabiston DC, Spencer FC. Gibbon's surgery of the chest. Philadelphia: WB Saunders, 1983; 733-772.
6. Payne WS, Ellis FH. Esophagus and diaphragmatic hernias. In: Schwartz SI, Shires GT, Spencer FI, Storer EH. Principles of surgey. McGraw-Hill, 1984; 1087-1090.

7. Aggerholm K, Illum P. Surgical treatment of Zenker's diverticulum. J Laryngol Otology 1990; 104: 312-4.

8. Zaninotto G, Costantini M, Boccu C, Anselmino M, Parenti A, Guidolin D, Ancona E. Functional and morphological study of the cricopharyngeal muscle in patients with Zenker's diverticulum. Br J Surg 1996; 83:1263-7

9. Westrin KM, Ergun S, Carlsoo B. Zenker's diverticulum: a historical review and trends in therapy. Acta Otolaryngol 1996; 116: 351-360.

10. Chin E, Coyle WJ. Zenker's diverticulum. Am J Gastroenterol 1997; 92 : 720 
11. Ludlow A. A case of obstructed deglution from a preternatural dilatation of a bag formed in the pharynx. Medical observations and inquieries $1769 ; 3$ : 85-101.

12. Zenker FA, Von Ziemssen H. Krankheiten des oesophagus. En: Von Ziemssen, H. (ed): Handbuch der speciellen pathologie und therapie. Vol. 7, Parte I (Suppl.) FCW Vogel, Leipzig 1874.

13. Chiquillo Barber MT, Ruiz del Castillo J, Bernal Sprekelsen JC, Mendoza Aroca A, Esclapez Valero JP, Martínez Agulló A et al. Tratamiento quirúrgico del divertículo de Zenker. A propósito de 14 casos. Rev Esp Enferm Dig 1990; 77: 251-3.

14. Wheeler WJ. Pharyngocele and dilation of pharynx with existing diverticulum at lower portion of pharynx lyng posterior to the oesophagus, cured by pharyngotomy, being the first case of the kind recorded. The Dublin Journal of Medical Science 1886; 82: 349-356.

15. Cherveniakov A, Tzekov C, Grigorov GE, Cherveniakov P. Acquired benign esophago-airway fistulas. Eur J Cardiothorac Surg 1996; 10: 713-6.

16. Von Doersten PG, Byl FM. Endoscopic Zenker's diverticulotomy (Dohlman procedure): forty cases reviewed. Otolaryngol Head Neck Surg 1997; 116: 209-12.

17. Lippert BM, Folz BJ, Gottschlich S, Werner JA. Microendoscopic treatment of the hypopharyngeal diverticulum with the CO2 laser. Lasers Surg Med 1997; 20: 394-401.

18. Koay CB, Bates GJ. Endoscopic stapling diverticulotomy for pharyngeal pouch. Clin Otolaryngol 1996; 21: 371-6.

19. Scher RL, Richtsmeier WJ. Endoscopic staple-assisted esophagodiverticulostomy for Zenker's diverticulum. Laryngoscope 1996; 106: 951-6.

20. Payne WS, King RM. Pharyngoesophageal (Zenker's) diverticulum. Surgical clinics of Northamerica 1983; 63: 815-24.

21. Payne WS, Pairolero PC, Piehler JM. The management of Zenker's diverticulum: Diverticulectomy. In: Kittle CF. Current controversies in thoracic surgery. Philadelphia. WB Saunders, 1986: 3-9.

22. Little AG, Skinner DB. The management of Zenker's diverticulum: Cricopharyngeal miotomy and diverticulopexy. In: Kittle CF. Current controversies in thoracic surgery. Philadelphia WB Saunders, 1986; 21-27.

23. Arnal Monreal FM, Rodriguez Pérez H, Ghanime Saide G, Gómez Castro A. Carcinoma de divertículo faringo-esofágico. Rev Esp Enferm Digest 1984; 65: 531-3.

24. Parrilla P, Aguayo JL, Ramírez P, Ortíz A, Martínez de Haro F. Divertículos esofágicos. Cir Esp 1987; 41: 102-7.

25. Bowdler DA, Stell PM. Carcinoma arising in posterior pharyngeal pulsion diverticulum (Zenker's diverticulum). Br J Surg 1987; 74: 561-3.

26. Jorgensen Th, Martínez Ramos C, Núñez Peña JR, Sanz López R, Ruiz de Gopegui, Tamames ES. Carcinoma en divertículo de Zenker de larga evolución. Rev Esp Enferm Digest 1994; 85: 203-7.

27. Halstead AE. Diverticula of the oesophagus. Ann Surg 1904; 39: 171-93.

28. Vinson PP. Simultaneous occurrence of multiple lesions in the oesophagus. Arch Otolaryngol 1927: 5: 502-6.

29. Wychulis AR, Gunnlaugsson GH, Clagett OT. Carcinoma occurring in pharyngoesophageal diverticulum. Surgery 1969; 66: 976-9.

30. Hoover WB. Carcinoma associated with esophageal diverticulum. Surg Clin North Am 1945; 25: 707-12.

31. Bertelsen S, Aasted A. Results of operative treatment of hypopharyngeal diverticulum. Thorax 1976; 31: 544-7.

32. Pierce WS, Johnson J. Squamous cell carcinoma arising in a pharyngoesophageal diverticulum. Cancer 1969; 24: 1068-1070.

33. Turner JM, Chir B. Carcinoma as a complication of pharyngeal pouch. Br J radiol 1963; 36: 206-210.

34. Nansom EM. Carcinoma in a long-standing pharyngeal diverticulum. Br J Surg 1976; 63: 417-419.

35. Rojas FAB, Szymanowsky RT, Fujita S. Zenker's diverticulum. Carcinoma of the oesphagus. J Otolaryngol 1979; 8: 266-70.

36. Zonnevylle JA, Waldeck KJJ, Hamming JJ. Carcinoma in situ in a pharyngo-esophageal diverticulum. Neth J Surg 1981; 33: 94-7.

37. Som ML, Deitel M. Carcinoma in a large pharyngo-esophageal diverticulum. Arch Surg 1967; 94: 35-38.

38. Lieberson M, Riese KT. Carcinoma in a large pharyngo-esophageal diverticulum. Gastroenterology 1960; 38: 817-20.

39. Bullok WK, Snyder EN. Carcinoma in situ occurring in a pharyngeal diverticulum. Cancer 1952; 5: 737-9.

40. Baraka ME, Sadek SAA. Carcinomatous change in pharyngeal diverticula. J Laryngol 1985; 95: 297-9.

41. Carcinoma "in situ" de un divertículo faringoesofágico (Divertículo de Zenker). R Burgos, G Tellez, E Ferrero, C Montero, M Gercia Font, I Vegazo et al. Cir Esp 1982; 36: 381-3.

42. Fisher MJ, Bond JF. Carcinoma in pharyngoesophageal diverticulum. J Thorac Cardiovasc Surg 1967; 53: 500-3.

43. Donald PJ, Huffman DI. Carcinoma in a Zenker's diverticulum. Head Neck Surg 1979; 2: 71-5.

44. Mackay IS. The treatment of pharyngeal poucy. J Laryngol Otol 1976; 90: $183-90$.

45. Jonhson JT, Curtin HD. Carcinoma associated with Zenker's diverticulum. Ann Otol Rhinol Laryngol 1985; 94: 324-5.

46. Garlock JH, Richter KT. Carcinoma in a pharyngo-esophageal diverticulum. Ann Surg 1961; 154: 259-62. 